ROMINA CAYUMIL MONTENCINO

FACULTAD DE INGENIERÍA

UNIVERSIDAD ANDRÉS BELLO

SANTIAGO, CHILE

ROMINA.CAYUMIL@UNAB.CL

MARCELO ADASME VALDÉS

FACULTAD DE INGENIERÍA

UNIVERSIDAD ANDRÉS BELLO

SANTIAGO, CHILE

M.ADASMEVALDS@UANDRESBELLO.EDU

Financiamiento: Financiado por Proyectos

Regulares de Investigación con Financiamiento

UNAB, año 2018, DI-20-18/"REG",

Universidad Andrés Bello, Chile.

Fecha de recepción: 19/06/2017

Fecha de aceptación: 26/08/2017

Cómo citar: Cayumil Montecino, R. y Adasme

Valdés, M. (2018), Desafíos y estrategias de

mejora en el manejo y procesamiento de

e-waste. RChD: creación y pensamiento, 3(5), 1-12

DoI: 10.5354/0719-837X.2018.49916

Revista Chilena de Diseño,

RChD: creación y pensamiento

Universidad de Chile

2018, 3(5)

http://rchd.uchile.cl

\section{Desafíos y estrategias de mejora en el manejo y procesamiento de $e$-waste}

\author{
Challenges and improvement strategies on e-waste \\ management and processing
}

Resumen. El incremento del uso de aparatos eléctricos y electrónicos (AEE), ha provocado la generación de grandes volúmenes de $e$-waste a escala global. Dichos residuos son los que más rápidamente aumentan en el mundo, se incrementan de 3 a $4 \%$ por año. Se estima que la generación global de e-waste durante el 2018 será de 49,8 millones de toneladas (Mt), de los cuales solamente un $20 \%$ sería formalmente procesado. Existen ciertos factores que influyen directamente en la generación de e-waste, tales como la obsolescencia programada, avances tecnológicos, y necesidad de recambio por equipos más nuevos, rápidos, y con características mejoradas.

Este artículo reporta la problemática de la generación de e-waste a nivel mundial, y alternativas de mejora para minimizar el impacto medioambiental asociado a su disposición. Dentro de la problemática, se indican volúmenes, complejidad inherente de la composición de los AEE, y consecuentes limitaciones en el manejo de los e-waste. Las alternativas para abordar dicha problemática se encuentran dentro del concepto de economía circular, que considera no solamente el producto final, sino que también el diseño, proceso e impactos relacionados a la manufactura. Dichas alternativas van desde el ecodiseño y cambios en la fabricación de AEE, hasta procesos sustentables de reciclaje una vez que estos alcancen su vida útil, incluyendo la sustitución de materiales en el proceso de manufactura por sustancias medioambientalmente más amigables.

Palabras clave: economía circular, e-waste, obsolescencia, reciclaje, sustentabilidad.

Abstract. The rise of the use of electric and electronic equipment (EEE), has resulted in the increase of the quantities of electronic waste (e-waste) generated worldwide. These solid residues are the fastest waste stream worldwide, increasing 3 to $4 \%$ annually. It has been estimated that in 2018, $49.8 \mathrm{Mt}$ will be produced globally, however, only $20 \%$ would be formally processed. There are several factors that directly influence on the e-waste generation, such as the programmed obsolescence, technology advances, and the need of newer, faster and updated equipment.

This article reports problems related to the e-waste generation worldwide, and improvement alternatives to minimize the environmental impact associated to the e-waste disposal. Among the problems, amounts of e-waste generated globally and regionally are presented, and the inherent complexity related to the EEE composition, and consequent limitations in the e-waste management. The alternatives to address these issues are based on the concept of circular economy, that considers not only the final product, but also the design, process and impacts related to the manufacture. Those alternatives include the green product design and changes on the manufacture of $\mathrm{EEE}$, and sustainable recycling processes once the EEE reach their end-of-life, including the materials substitution during the manufacturing process by using environmentally friendly substances.

Keywords: circular economy, e-waste, obsolescence, recycling, sustainability. 


\section{Introducción}

El efecto de la globalización y el exponencial crecimiento demográfico han promovido el aumento del uso de aparatos eléctricos y electrónicos (AEE). Estos aparatos son utilizados en un amplio rango de aplicaciones. En la actualidad, los AEE son cada vez más accesibles, dada la gran oferta y necesidades de la población. El incremento del uso de AEE tiene como consecuencia la generación de grandes volúmenes de residuos de aparatos eléctricos y electrónicos (RAEE o e-waste, por su sigla en inglés). Dichos residuos son los que más rápidamente aumentan en el mundo, sus volúmenes se incrementan de 3 a $4 \%$ por año. La generación global de e-waste en 2016 fue de 44,7 Mt, y se estima que el 2018 se producirán $49,8 \mathrm{Mt}$ (millones de toneladas) a nivel mundial (Baldé et al., 2017).

De los e-waste generados en 2016, solo 8,94 Mt fueron formalmente recolectados y procesados, lo que corresponde al $20 \%$ de la generación global anual. Esta cifra está directamente relacionada a países que implementaron regulaciones internas sobre el manejo y tratamiento de e-waste (Baldé et al., 2017). En las últimas décadas, la gestión de los e-waste se ha convertido en un tema de relevancia mundial. Ha habido grandes avances en la implementación de normativas de manejo y disposición de e-waste, aún la gran mayoría de los países no tiene regulaciones para este tipo de residuos (Khanna et al., 2018). Algunas de las limitaciones en la implementación de dichas normativas son la recolección y la restricción de las regulaciones a solamente algunos tipos de e-waste. Dichas limitaciones, sumado a la falta de regulaciones, hace que su procesamiento sea difícil. Como resultado de esto hay un enorme impacto en el ambiente, dado que los e-waste aún son dispuestos junto con los residuos domésticos, por lo que contaminan el medio ambiente, y pierden recursos que pudiesen ser recuperados y reutilizados. Por otro lado, estos contienen compuestos potencialmente tóxicos, por lo que la disposición inapropiada de estos implica la liberación de dichas sustancias, y provocar altos niveles de contaminación. Sin embargo, durante los últimos años, se ha generado conciencia acerca del impacto medioambiental que genera la disposición y procesamiento inapropiado de e-waste. La complejidad inherente en la composición de los AEE y consecuentes limitaciones en el manejo de los e-waste hacen que su reciclaje sea un desafío (Moreno et al., 2014; Preston, 2012). En este artículo se investigarán las alternativas existentes para minimizar la complejidad existente en la gestión y procesamiento de los e-waste.

El objetivo de este estudio es presentar la problemática y los factores asociados a la generación de e-waste a nivel mundial, y alternativas de mejora en el manejo de estos una vez alcanzada su vida útil, tales como en la fabricación de $A E E$, diseño, sustitución de materiales y procesos de reciclaje, lo cual se enmarca dentro del concepto de economía circular.

\section{Problemática asociada a los e-waste}

\subsection{Generación de e-waste}

La generación de e-waste a nivel mundial el año 2016 fue de 44,7 Mt, lo cual es equivalente a $6,1 \mathrm{~kg} / \mathrm{hab}$. Se estima que para el año 2021 , el volumen de e-waste generado globalmente aumente a 52,2 Mt, correspondiente a 6,8 kg/ hab. Dichos volúmenes tienen un crecimiento anual de 3 a $4 \%$ (Baldé, et al., 2017). En la Figura 1 se presentan los volúmenes de $e$-waste producidos el año 2016 por continente, se indica la generación per cápita por región. 

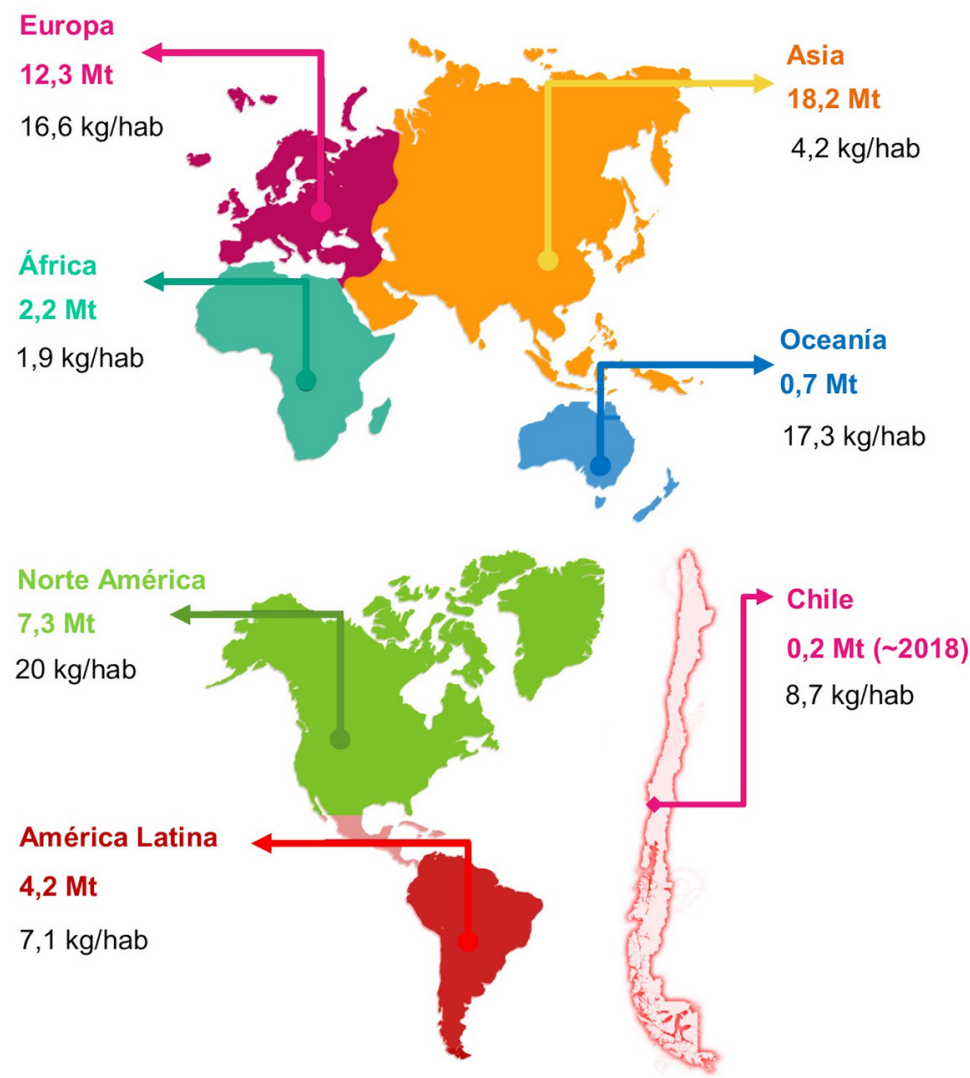

El mayor generador de e-waste por continente en 2016 fue Asia con 18,2 Mt, seguido por Europa con 12,3 Mt, y América con 11,3 Mt. Los continentes que menos e-waste generaron fueron África y Oceanía, con 2,2 y o,7 Mt, respectivamente. En cuanto a regiones con mayor generación per cápita, se tiene a Oceanía y Europa con $17,3 \mathrm{~kg} / \mathrm{hab}$ y $16,6 \mathrm{~kg} / \mathrm{hab}$, respectivamente (Baldé, et al., 2017). Con respecto a la situación en América, en 2016 se generó el $25 \%$ del volumen de e-waste producido a nivel mundial, con un promedio de $11,6 \mathrm{~kg} / \mathrm{hab}$. Sin embargo, solo en Norteamérica se generan $20 \mathrm{~kg} / \mathrm{hab}$., que es donde se concentra la mayor generación de $e$-waste del continente. El mayor generador de e-waste por país es Estados Unidos con 6,3 Mt, en segundo lugar, Brasil con 1,5 Mt, y tercero, México con I Mt (Baldé, et al., 2017).

En América Latina, la generación de e-waste en el año 2016 fue de 4,2 Mt, con un promedio de $7,1 \mathrm{~kg} / \mathrm{hab}$. Como se mencionó anteriormente, la tasa de aumento del volumen de e-waste a nivel mundial es de 3-4\%, sin embargo, el crecimiento estimado en Latinoamérica al 2018 es de $5 \%$ (Magalini et al., 2015). Se ha estimado que Chile producirá alrededor 200,000 Mt en 2018. A pesar de que esta cantidad es mucho menor que lo que genera Brasil y México, Chile es el segundo mayor productor de e-waste per cápita en América Latina. Uruguay lidera dicha generación con $10,8 \mathrm{~kg} / \mathrm{hab}$, seguido por Chile con $8,7 \mathrm{~kg} / \mathrm{hab}$ (Magalini et al., 2015).
Figura 1. Volúmenes de e-waste generados por región (Baldé, et al., 2017; Magalini et al., 2015). 


\subsection{Características de los e-waste y sus impactos}

Los asperctos asociados a la generación de e-waste son múltiples. Primeramente, los e-waste tienen una composición altamente compleja. Más de 1.000 sustancias están presentes en un amplio rango de AEE (Cui \& Zhang, 2008). Esta composición heterogénea incluye tanto elementos valiosos como sustancias peligrosas y potencialmente tóxicas. Las placas madre, componente central de los AEE, contienen hasta 40\% de metales y alrededor de $60 \%$ de no metales, tales como polímeros, resinas, fibra de vidrio, cerámicos, entre otros. (Kasper et al., 2011).

La composición de los AEE depende de varios factores, tales como el tipo, función, peso, edad y fabricante. Adicionalmente, nuevos productos han sido puestos en el mercado, los cuales tienen nuevas funciones y características. Sumado a eso, la composición de un componente en específico puede ser diferente de otro. Un clásico ejemplo son las placas madre, componente fundamental de los AEE. Para cumplir con el rendimiento requerido del equipo, existen distintos tipos de placas madre, y la selección de materiales depende fuertemente de su función y diseño.

Debido a que la industria electrónica usa grandes cantidades de metales, tales como cobre y metales preciosos, dichos metales son un recurso valioso presente en los e-waste (Cayumil R., 2017). La concentración de cobre presente en los e-waste puede encontrarse entre el 10 y $20 \%$ del peso total del equipo (Cui \& Roven, 2011; Kogan, 2006; Schluep et al., 2009), concentración que es significativamente mayor que la que se encuentra en el cobre mineral, el cual contiene entre $0,5 \%$ y $1 \%$ de $\mathrm{Cu}$ (Schlesinger et al., 2011). Similarmente, la concentración de metales preciosos, tales como plata, oro, platino y paladio es también mayor en placas madre que en sus respectivos minerales (Hagelüken, 2006). El valor del cobre y metales preciosos corresponden entre el 40 y $70 \%$ del total del valor del equipo en desuso (Cui \& Roven, 2011). En consecuencia, estos metales preciosos, más la presencia de tierras raras, permiten estimar un potencial valor económico en los e-waste generados en 2016, de 55 billones de euros (Baldé et al., 2017).

Las altas cantidades de metales valiosos presentes en los e-waste ha repercutido en el desarrollo de reciclaje informal de e-waste, donde se recuperan metales de manera inapropiada e insegura. Son prácticas no certificadas, realizadas a pequeña escala y cercanas a los asentamientos urbanos o aledañas al vertedero de electrónicos (Robinson, 2009).

Este reciclaje informal genera residuos y emisiones potencialmente tóxicas y peligrosas, que contaminan no solo el suelo, sino que también el aire y el agua, lo cual afecta a la población mediante la cadena alimenticia. Algunas sustancias que se liberan al medio ambiente debido al mal procesamiento son el plomo, mercurio, cadmio, cromo, y los contaminantes orgánicos persistentes (COPS), entre los cuales se encuentran los bifenilos policlorados (PCB) y las dioxinas y furanos, entre otros (Robinson, 2009).

Otro factor relevante que influye en la alta cantidad de e-waste generado a nivel mundial, es la obsolescencia programada de los AEE puestos en el mercado. Los equipos son diseñados y programados para tener una vida útil específica, lo cual obliga al usuario a renovar aparatos electrónicos regularmente (Moreno, 2014). Sin embargo, los consumidores no solo reemplazan equipos debido a la falla de un artículo, sino que también por la necesidad de obtener un nuevo equipo que atienda sus necesidades (Preston, 2012).

La vida útil de los AEE depende fuertemente del tipo de equipo. Por ejemplo, 
la probabilidad de descartar un computador portátil en menos de cinco años de uso es de alrededor de un $11 \%$, siendo uno de los más altos dentro de IOS AEE. El descarte de equipos en el corto plazo se debe a la sofisticación y mejora continua de tecnologías, como es el caso de smartphones y laptops. En contraste, existen otros AEE cuya vida útil es mucho mayor, tales como las lavadoras y los acondicionadores de aire (Baldé et al., 2017).

\section{Estrategias de mejora en el manejo de e-waste}

\subsection{Nuevas tendencias en diseño}

Dentro de los cambios que han sido incorporados en el diseño y fabricación de AEE, uno de ellos es la utilización de procesos más sustentables, eficientes, con menos generación de residuos, y con la utilización de materiales reciclados y reciclables, además de minimizar la contaminación cuando termine su vida útil (Ciocci \& Pecht, 2006). Algunas propuestas implementadas alrededor del mundo son la Responsabilidad Extendida del Productor (REP), ley de etiquetado, administración de productos, reciclaje, remanufactura, ecodiseño de productos, y diseño para el medio ambiente (Herat, 2007). El concepto relacionado con la mejora en el diseño de los AEE, es el ecodiseño de productos ( $D f E$, por su sigla en inglés), cuyo propósito es utilizar materiales en la fabricación del dispositivo que reduzcan el riesgo de contaminación, una vez terminada su vida útil (Zhang et al., 1997). Los factores que se desarrollan en esta etapa son la eliminación de productos tóxicos, mejor desensamblaje, menor peso y menor huella ecológica. Estas nuevas características en diseño son además una incorporación de ventajas competitivas entre fabricantes (Herat, 2007).

Dado lo anterior, es de esperar que los consumidores prefieran productos que tengan las siguientes características: que el equipo pueda ser retirado una vez terminada su vida útil, que contenga menos cantidad de compuestos tóxicos, fabricado con una mayor cantidad de materiales reciclados, fácil de desensamblar, con alta eficiencia energética, pocos y más sustentables materiales de embalaje, y con certificación de producto ambientalmente sustentable (Herat, 2007). El hecho de incentivar prácticas ecológicas no busca restringir el uso de tecnologías, sino que extender la vida útil de los AEE (Cooper, 2010). Un caso representativo de un dispositivo diseñado de tal forma que permite actualizar piezas de manera independiente, es el "Fairphone 2", un dispositivo móvil inteligente. A este tipo de móviles se les conoce como "Smartphone Modular" (Fairphone, 2018). Entre sus características, se encuentran la rápida puesta en operación luego de un accidente, o falla de alguna parte interior, lo que extiende la vida útil del aparato. Otro "Smartphone Modular" en el mercado es el "LG G5" y "LG G5 SE", que tiene características más lujosas (LG Electronics, 2018).

Se genera un impacto positivo en el diseño, fabricación y ciclo de vida del producto, a través de la utilización de estos dispositivos, reduciéndose la generación de e-waste, ya que es únicamente procesada la pieza averiada, y, al mismo tiempo, vincula a los consumidores (Fairphone, 2018).

\subsection{Reemplazo de materiales}

Con el fin de minimizar la generación de e-waste, y consecuente impacto medioambiental debido a su disposición, el reemplazo de sustancias peligrosas por materiales menos contaminantes utilizados en la fabricación de AEE, se torna fundamental al momento de generar una estrategia de reducción, 
tanto de volúmenes de e-waste dispuestos, como de dicho impacto ambiental. Estos materiales alternativos son usados en las diferentes etapas de manufactura de un amplio rango de AEE (Zhang et al., 1997).

Debido a la toxicidad y persistencia en el medio ambiente, se ha prohibido la utilización e incluso la fabricación de ciertos compuestos y elementos, tales como los contaminantes orgánicos persistentes, firmado en la Convención de Estocolmo. Los contaminantes orgánicos persistentes (COPS) son compuestos tóxicos, resistentes a la degradación y bioacumulativos. Se acumulan en el aire, agua y en especies migratorias, por lo que pueden ser transportados y depositados en lugares diferentes de donde fueron generados, y, además, se acumulan en ecosistemas terrestres y acuáticos (Harrad, 2010). De acuerdo a la Convención de Estocolmo, dentro de los cops que han sido prohibidos, se pueden encontrar pesticidas, químicos industriales, tales como los bifenilos policlorados (PCBS, por su sigla en inglés), y subproductos del procesamiento de ciertos compuestos, como por ejemplo dioxinas y furanos policlorados (PCDD/Fs.) (United Nations, 2018). Además son potencialmente cancerígenos (Wright, 2003).

En el caso particular de la fabricación de AEE, ha habido una evolución en la composición de ciertos componentes presentes en estos aparatos. Ciertas sustancias han sido reemplazadas por compuestos más sustentables, para evitar la contaminación asociada a materiales potencialmente tóxicos o contaminantes. La variación en la composición no solo se realiza para evitar la contaminación, sino que también tiene relación con los cambios en la función de ciertos componentes, antigüedad en la fabricación, y fabricante. Por otro lado, la finita disponibilidad de ciertos recursos metálicos también ha influido cambios en los materiales utilizados en la manufactura de AEE. Algunos casos que serán expuestos, son la soldadura de placas madre, plomo presente en pantallas y monitores, y gases refrigerantes. Las placas madre son un claro ejemplo de la evolución en la utilización de algunos elementos en la fabricación de AEE. La soldadura presente en las placas es utilizada para conectar varios componentes electrónicos en la superficie de la placa, y puede contener plomo, estaño, plata y cadmio. La mayoría de los aparatos antiguos contiene soldadura plomo-estaño, que generalmente es de composición $37 \% \mathrm{~Pb}$ y $63 \% \mathrm{Sn}$ (Bizzo et al., 2014). En los últimos años, se ha restringido el uso de plomo en las soldaduras, sin embargo, una gran cantidad de placas madre obsoletas contienen plomo, lo cual es considerado un desafío en el procesamiento de ese material. No obstante, nuevos procesos de fabricación usan aleaciones metálicas libres de plomo en placas madre. Estas aleaciones son del tipo estaño-plata-cobre como primera opción, con la combinación de otras aleaciones como estaño-zinc-bismuto, estaño-cobre y estaño-bismuto-plata (Suganuma, 2001). Los gases refrigerantes presentes en refrigeradores, aire acondicionado y congeladores son otros casos de cambios en la composición de AEE a través del tiempo. Los gases refrigerantes son aquellos que se encuentran en todos los sistemas de refrigeración, y que sirven para regular la temperatura del entorno debido a sus propiedades fisicoquímicas. El uso de este tipo de sistemas ha incrementado con la variación de temperatura en ciertas regiones del planeta y el crecimiento demográfico que afecta directamente la intensidad de uso de estos aparatos electrónicos. Dentro de los gases refrigerantes, se destacan tres tipos de compuestos: cloro-fluorocarbonos (CFC), hidro-cloro-fluorocarbonos (HCFC) e hidro-fluorocarbonos (HFC), que son conocidos como refrigerantes 
fluorados, de naturaleza peligrosa (Benhadid-Dib \& Benzaoui, 2012).

Estos compuestos son gaseosos a temperatura ambiente, poseen baja solubilidad en el agua, y tienen alta estabilidad química, tienen una vida estable entre 10 y 400 años, dependiendo de la complejidad de la molécula (Wright, 2003). Es por ello que, al ser emitidos a la atmósfera, pueden mezclarse con otros gases y dispersarse sin límite hasta llegar a la capa de ozono, lo que contribuye al efecto invernadero y a su vez actúan como sustitutos de la capa de ozono, absorbiendo el calor atmosférico y devolviéndolo a la tierra. Se tienen registros de las emisiones solo cuando los AEE son desechados. Estas emisiones existen desde el descubrimiento del gas en 1930 (McCulloch et al., 2002; U.S. National Library of Medicine, 2018).

Los HFC son menos estables que los CFCS, tienen una duración de diez años y se denominan sustancias de transición. De esta manera, se ha producido un reemplazo gradual de CFCS y HCFCS por HFCS, ya que estos no contienen cloro, por lo que son menos persistentes. Dicha sustitución ha sido promovida por el Protocolo de Montreal, al cual Chile se integró en 1987 (Dto 238, Ministerio de Relaciones Exteriores, 2018; Cayumil et al., 2016).

En el año 2013, en Chile se implementó un proyecto llamado "Plan de Gestión para la Eliminación de HCFCs de Chile" que integraba recursos públicos e inversiones privadas para obtener tecnologías extranjeras no agotadoras de la capa de ozono, con el fin de utilizar gases refrigerantes de bajo o nulo impacto ambiental. Se fijó la eliminación del $97 \%$ de HCFCS o CFCS al 2030. Además, el proyecto integra un subsidio de equipos refrigerantes para Pymes y empresas de mayor escala (Dto 146, Ministerio de Relaciones Exteriores, 2018; Ministerio del Medio Ambiente, 2018).

Otra sustitución importante es la utilización de $\mathrm{CO}_{2}$ como gas refrigerante con mínimos impactos ambientales, y que además favorece la eficiencia energética de los sistemas refrigerantes, disminuyendo la huella de carbono que generan las centrales hidroeléctricas (United Nations Environment Programme, 2002).

\subsection{Procesos de reciclaje}

El reciclaje una vez terminada su vida útil es una de las soluciones a la disposición de e-waste, y consecuente liberación de sustancias tóxicas. El reciclaje debe estar planificado en la etapa de diseño y fabricación del equipo, en el cual el tipo de materiales utilizados, proceso de desensamblaje, entre otros factores, juegan un rol fundamental a la hora de procesar los e-waste (Cooper, 2010; Preston, 2012). Los procesos de reciclaje incluyen recuperación tanto de sustancias no metálicas como metálicas. Esta sección se enfocará en la recuperación de metales desde $e$-waste.

La cadena de reciclaje es un proceso que consta de cuatro etapas consecutivas: recolección, desmantelamiento o desensamblaje, preprocesamiento, y procesamiento o recuperación de materiales (Schluep et al., 2009; Tsydenova \& Bengtsson, 2011). La última etapa, también llamada procesamiento metalúrgico, o proceso de reciclaje, es el encargado de efectivamente recuperar metales para darle un nuevo uso (Cui \& Roven, 2011; Cui \& Zhang, 2008). Los procesos de recuperación generalmente utilizan tratamientos hidrometalúrgicos y pirometalúrgicos, con procesamiento electrometalúrgico posterior. La hidrometalurgia consiste en la disolución de materiales en soluciones con ácido, cianuro, haluros, entre otros, seguido de una etapa de purificación/concentración del elemento de interés. Por otro lado, la pirometalurgia es un proceso que utiliza altas temperaturas para separar metales 
e impurezas, que tiene como propósito fundir los e-waste para formar dos fases: metálica, que contiene metales, tales como cobre, oro, plata, entre otros, y no metálica, compuesta por óxidos y/o cerámicos. El proceso final es electrorefinación o electroobtención, donde se recuperan los elementos metálicos (Cui \& Zhang, 2008; Kim et al., 2009; Robinson, 2009).

A pesar de que existen algunos procesos industriales que tratan placas madre (dadas sus altas cantidades de elementos valiosos), ninguno de estos es un proceso específico para e-waste, sino que dichas placas se procesan en conjunto con otro tipo de residuos, o con concentrados de cobre. Como la composición de los e-waste es altamente compleja, y radicalmente diferente de minerales, muchas de las investigaciones actuales en el reciclaje de e-waste se han enfocado en la recuperación de cobre y metales preciosos, donde se utilizan tanto hidro como pirometalurgia, con diversas condiciones de proceso. Si bien con las investigaciones se han obtenido resultados relevantes, aún se requieren mejoras en los procesos.

Se ha reportado la creación de un proceso sustentable para recuperar metales y no metales desde e-waste, que minimiza las emisiones de gases contaminantes, y producción de residuos sólidos. Este proceso se denomina pirólisis de alta temperatura, el cual concentra y recupera cobre y metales preciosos desde placas madre. Al trabajar con una atmósfera inerte, además se genera un producto rico en carbón, dado que este se mantiene en estado sólido, para evitar la formación de gases de efecto invernadero, tales como co y CO2. La operación a temperaturas mayores que $800^{\circ} \mathrm{C}$, además de la atmósfera inerte, evita la generación de dioxinas y furanos. Dado que las temperaturas de operación son mucho menores que los procesos pirometalúrgicos tradicionales de recuperación de cobre $\left(1250^{\circ} \mathrm{C}\right)$, este proceso es energéticamente eficiente. Este método es sustentable con el medio ambiente, y produce productos metálicos y no-metálicos, que pueden ocuparse como recursos (Cayumil et al., 2014; Cayumil et al., 2018; Cayumil et al., 2016; Sahajwalla et al., 2015).

\section{Ley Responsabilidad Extendida del Productor}

Esta ley involucra a todos los AEE, donde son relevantes las cualidades de sus componentes químicos, categorizando a los aparatos según su vida útil y su potencial toxicidad. Así, surge la Ley REP (de Responsabilidad Extendida del Productor), para el control de los aparatos más tóxicos y de corta vida útil, lo que regulariza la producción de los aparatos, por medio de normas que involucran directamente a los fabricantes, por lo tanto, el que produce contaminantes debe pagar por los contaminantes que ofrece, aun cuando la vida útil del aparato haya caducado, dado que a su vez el productor adquiere un beneficio económico con la venta de los AEE (Duque, 2018). La responsabilidad extendida del productor en Chile fue promulgada el 2016, junto con el marco para la gestión de residuos y la Ley de Fomento al Reciclaje. Esta última tiene por objetivo incorporar valor económico a los residuos, como una base para la gestión de su reciclaje, favorecido por la ley que castiga los depósitos ilegales de e-waste. Entonces, el productor se hace cargo de los e-waste, al registrar, organizar y financiar las metas de recolección, lo que asegura además el tratamiento de los residuos recolectados. De esta manera, se establecen los principios propuestos por el Ministerio de Medio Ambiente: "el que contamina paga", "responsabilidad del creador del residuo", "transparencia y publicidad" y el más importante "trazabilidad" en 
la gestión del residuo. El no cumplir con la ley 19.200 en cuestión, arriesga sanción escrita y multa de hasta 10.000 unidades tributarias anuales, las cuales se calcularán en función del beneficio económico que el productor haya obtenido por la infracción propiamente tal (Ley 20.920, Ministerio de Medio Ambiente, 2018).

\section{Análisis}

Tanto el agotamiento de los recursos naturales, como la creciente preocupación por la contaminación y generación de residuos a nivel mundial, ha impulsado el sistema de economía circular, que en el proceso productivo considera no solo el producto, sino que también el diseño de los aparatos, los procesos de manufactura, y los impactos ambientales asociados a dichos procesos. Un caso particularmente complejo es el de los e-waste. Se han presentado diversos aspectos de la problemática asociada a la generación de e-waste a nivel mundial para resolver dichos problemas, varias estrategias de mejora en su manejo han sido reportadas. Dentro de dichas estrategias, la de ecodiseño cobra especial relevancia, dado que se encuentra al inicio de la cadena productiva de dispositivos electrónicos, y tiene grandes consecuencias positivas una vez finalizada la vida útil de los aparatos. Dentro de sus características, se encuentran la minimización de la complejidad en el manejo de e-waste, lo cual impacta directamente en el procesamiento de los dispositivos una vez finalizada su vida útil (Kasper et al., 2011; Cooper, 2010).

Gracias a las alternativas de ecodiseño, la implementación de legislaciones de gestión de e-waste, y el constante aumento de los volúmenes de residuos generados, es que los procesos de reciclaje resultan técnicamente factibles. Los procesos de minería urbana para la valorización de e-waste cobran fuerza, con los que es posible recuperar tanto de materiales valiosos desde $e$-waste como minimizar los efectos negativos relacionados a la disposición de AEE en desuso y al procesamiento informal e inseguro de los mismos.

Es importante destacar que el consumidor juega un rol fundamental en los tipos de AEE adquiridos, y por lo tanto en el $e$-waste generado. Se puede utilizar el marketing como una herramienta de incentivo proecológico, por ejemplo, en el uso de smartphones modulares (Kotler \& Keller, 2012).

En el caso de Chile, dado que los fabricantes de AEE no son locales, no se puede hacer ecodiseño de dispositivos. Sin embargo, se pueden implementar técnicas de procesamiento de e-waste debido a la existencia de la ley REP que impulsa estas prácticas, así como también la oportunidad que se presenta de desarrollar minería urbana, en un país con larga experiencia en minería primaria.

\section{Conclusiones}

La creciente generación de e-waste a nivel mundial ha alertado a los gobiernos y la población en general a tomar medidas respecto del impacto medioambiental que genera la incorrecta disposición de estos residuos. Las altas cantidades de $e$-waste generados están directamente relacionados con los niveles de desarrollo de los países, y las necesidades de los usuarios. La obsolescencia programada de los AEE puestos en el mercado es uno de los factores principales asociados a los altos volúmenes de e-waste generados a nivel mundial. El diseño de los AEE está planificado para tener cierta vida útil. Otros factores que influyen en la generación de e-waste son los avances tecnológicos y la necesidad de tener aparatos más pequeños, rápidos y modernos. La combinación y tipos de materiales y compuestos usados en un rango de 
AEE han evolucionado dramáticamente a través del tiempo. Las proporciones relativas de las substancias usadas en la fabricación de AEE han variado debido a varios factores, tales como regulaciones en el uso de materiales peligrosos y elementos potencialmente tóxicos, nuevas funciones, y la necesidad de tener equipos más rápidos, pequeños y livianos.

El concepto de economía circular cobra real importancia al momento de entender las mejoras realizadas desde el diseño hasta el fin de su vida útil. El diseño de AEE debe atender tanto el manejo de residuos, como de emisiones y su disposición final. Actualmente, los fabricantes están eliminando el uso de elementos tóxicos, dadas las nuevas legislaciones como la Restricción de Sustancias Peligrosas en aparatos eléctricos y electrónicos, RoHS de la Unión Europea o mediante la iniciativa voluntaria de algunas empresas. Para implementar estos cambios, se han sustituido materiales en la fabricación de un alto rango de AEE, para reemplazar las sustancias peligrosas por compuestos medioambientalmente amigables y sustentables. Esto ha sido fundamental para enfrentar los nuevos requerimientos, tanto de los consumidores, como de las regulaciones y normativas.

Varias de las alternativas de mejora incluyen procesos más sustentables de fabricación de equipos, utilización de materiales menos tóxicos y menos contaminantes, componentes más fáciles de desensamblar y desarrollo de procesos de reciclaje para cuando estos aparatos alcancen su vida útil. Este proceso de mejora continua en la sustitución de materiales permite al corto y largo plazo diseñar procesos metalúrgicos para la extracción y refinación de metales, lo que podría ser en el futuro una economía competitiva (cuando ya la minería produzca más contaminantes que beneficios) (Wright, 2003), y poder así completar el "círculo", de una factible economía circular.

\section{Referencias}

Baldé, C., Forti, V., Gray, V., Kuehr, R., \& Stegmann, P. (2017). The Global E-waste Monitor. Bonn/ Geneva/Vienna: United Nations University (UNU), International Telecommunication Union (ITU) \& International Solid Waste Association (ISWA).

Benhadid-Dib, S., \& Benzaoui, A. (2012). Refrigerants and their environmental impact Substitution of hydrochlorofluorocarbon HCFC and HFC hydrofluorocarbon. Search for an adequate refrigerant. Energy Procedia, 807-816.

Bizzo, W., Figueiredo, R., \& de Andrade, V. (2014). Characterization of printed circuit boards for metal and energy recovery after milling and mechanical separation. Materials, vol. 7, n. 6, 4555-4566.

Cayumil, R. (2017). Minería Urbana: Reciclaje de desechos electrónicos para la recuperación de materiales valiosos. (S. M. Bravo, Ed.) In Cuadernos de ciencias y tecnologías de la tierra, 137-147.

Cayumil, R., Ikram-Ul-Hap, M., Khanna, R., Saini , R., Mukherjee, P., Mishra, B., \& Sahajwalla, V. (2018). High temperature investigations on optimising the recovery of copper from waste printed circuit boards.
Waste Management, Vol. 73, pp. 556-565.

Cayumil, R., Khanna, R., Ikram-Ul-Haq, M., Rajarao, R., Hill, A., \& Sahajwalla, V. (2014). Generation of copper rich metallic phases from waste printed circuit boards. Waste Management, 1783-1792.

Cayumil, R., Khanna, R., Rajarao, R., Ikram-ul-Haq, M., Mukherjee, P., \& Sahajwalla, V. (2016). Environmental Impact of Processing Electronic Waste - Key Issues and Challenges. INTECH. Obtenido de http://dx.doi. org/10.5772/64139

Cayumil, R., Khanna, R., Rajarao, R., Mukherjee, P., \& Sahajwalla, V. (2016). Concentration of precious metals during their recovery from electronic waste. Waste Management, vol. 57, 121-130.

Ciocci, R., \& Pecht, M. (2006). Impact of environmental regulations on green electronics manufacture. Microelectronics International, 23, 45-50. doi:10.1108/13565360610659716

Cooper, T. (2010). Longer Lasting Products. (T. Cooper, Ed.). England: Gower Publishing Limited.

Cui, J., \& Roven, H. (2011). Electronic Waste. In Waste: A handbook for management: Letcher. Academic Press, 281-296. 
Cui, J., \& Zhang, L. (2008). Metallurgical Recovery of Metals from Electronic Waste: a Review. Journal of Hazardous Materials, 158 (2-3), 228-256.

Directive, E.U. (2012). 2012/19/EU of the European parliament and of the council on waste electrical and electronic equipment. Official Journal of the European, 197, 38-71.

Dto 146, Ministerio de Relaciones Exteriores. (18 de Junio de 2018). Biblioteca del Congreso Nacional de Chile (BCN): Ley Chile. Obtenido de Promulga La Revisión Sustantiva $\mathrm{N}^{\circ} 3$ Del Acuerdo Con El Programa De Las Naciones Unidas para el Desarrollo Sobre El Proyecto: "Plan De Gestión Para La Eliminación De HCFC En Chile": https://www.leychile.cl/ Navegar?idNorma $=1112697$

Dto 238, Ministerio de Relaciones Exteriores. (18 de Junio de 2018). Promulga protocolo de montreal relativo a las sustancias agotadoras de la capa de ozono, de 16 de septiembre de 1987. Obtenido de Biblioteca del Congreso Nacional de Chile (BCN) - Ley Chile: http://bcn.cl/ıvobz

Duque, I. (2018). Asesoría para la implantación de la responsabilidad extendida del productor en Chile. Sector de aparatos eléctricos y electrónicos. My Global Lab Smart Projects.

Fairphone. (2018). Fairphone. Retrieved from https:// shop.fairphone.com/es/partes-de-repuesto

Gordon, R. B., Bertram, M., \& Graedel, T. E. (2006). Metal stocks and sustainability. Proceedings of the National Academy of Sciences, 103(5), 1209-1214. https://doi. org/10.1073/pnas.0509498103

Hagelüken, C. (2006). Recycling of electronic scrap at Umicore's integrated metals smelter and refinery. Erzmetall, vol. 59, n.3, 152-161.

Haig, S., Morrish, L., Morton, R., \& Wilkinson, S. (2012). Electrical product material composition. Waste and Resources Action Programme, Branbury, Oxon.

Harrad, S. (2010). Persistent Organic Pollutants. United Kingdom: WILEY.

Herat, S. (2007). Sustainable Management of Electronic Waste. WILEY-VCH, 305-310. doi:10.1002/ clen.200700022

Kasper, A., Berselli, G., Freitas, B., Tenório, J., Bernades, A., \& Veit , H. (2011). Printed wiring boards for mobile phones: Characterization and recycling of copper. Waste Management, Vol. 31, n.12, 2536-2545.

Khanna, R., Park, M., Cayumil, R., Mishra, S.K. \& Mukherjee, P.S. (2018) Global Perspectives on Regulatory Measures and Collection Procedures for Electronic Waste: $A$ Critical Assessment. Open Access Journal of Waste Management \& Xenobiotics, Vol. 1(1), pp. 000101

Kim, B., Lee, J., Jeong, J., \& Kang, S. (2009). A high temperature process for extracting valuable metals from waste electric and electronic scraps (WEES). Mater, vol. 50, n. 6, 1558-1562.

Kotler, P., \& Keller, K. (2012). Dirección de Marketing. Marketing (14th ed., Vol. 53). Mexico. https://doi. org/10.1017/CBO9781107415324.004

Ley 20.920, Ministerio de Medio Ambiente. (19 de Junio de 2018). Ley 20.920: Establece marco para la gestión de residuos, la responsabilidad extendida del productor y fomento al reciclaje. Obtenido de Biblioteca del Congreso Nacional de Chile (BCN) - Ley Chile: http:// bcn.cl/1vy5r

LG Electronics. (2018). LG G5 SE Titan - El Primer Smartphone Modular del Mundo. Retrieved from https://www.lg.com/cl/celulares/lg-G5-Titan-h840

Magalini, F., Kuehr, R., \& Baldé, C. (2015). eWaste in Latin America: Statistical analysis and policy recommendations. London and Bonn.

McCulloch, A., Midgley, P., \& Ashford, P. (2002). Releases of refrigerant gases (CFC-12, HCFC-22 and. Atmospheric Environment, 889-902.

Ministerio del Medio Ambiente. (18 de Junio de 2018). plan de gestión para la eliminación de HCFC de chile (HPMP). Valparaiso, Chile.

Moreno, M., Braithwaite, N., \& Cooper, T. (2014). Moving beyond the circular economy. In Proceedings of Going Green-CARE Innovation. (pp. 1-10). Australia. Retrieved from http://www.researchgate.net/ publication/281115672

Preston, F. (2012). A Global Redesign? Shaping the Circular Economy. Energy, Environment and Resource Governance, (March), 1-20. https://doi. org/10.1080/0034676042000253936

Robinson, B. H. (2009). E-waste: An assessment of global and environmental impacts. Science of the Total Environment, 183-191.

Sahajwalla, V., Cayumil, R., Khanna, R., Ikram-Ul-Haq, M., Rajarao, R., Mukherjee, P., \& Hill, A. (2015). Recycling polymer rich waste printed circuit boards at high temperatures: Recovery of value-added carbon resources. Journal of Sustainable Metallurgy, 75-84.

Schlesinger, M. E., King, M. J., Sole, K. C., \& Davenport, W. G. (2011). Extractive Metallurgy of Copper. Amsterdam: elsevier.

Schluep, M., Hagelueken, C., Magalini, R., Maurer, C., Meskers, C., Mueller, E., \& Wang, F. (2009). Recycling - From E-Waste to Resources. UNEP United Nations Environment Programme.

Suganuma, K. (2001). Advances in Lead-free Electronics Soldering. Current Opinion in Solid State and Materials Science, vol. 5, 55-64. doi:10.1016/S1359 
Tsydenova, O., \& Bengtsson, M. (2011). Chemical hazards associated with treatment of waste electrical and electronic equipment. Waste Manage, vol. 31, n. 1, 45-58.

U.S. National Library of Medicine. (18 de june de 2018). Tox Town: Developed by Specialized Information Services, Environmental Health and Toxicology. Obtenido de Clorofluorocarbonos (CFC): https://toxtown.nlm.nih. gov/espanol/chemicals. php?id $=40$

United Nations. (18 de June de 2018). Stockholm Convention: Protecting human health and the environment from persistent organic pollutants. Obtenido de http://chm.pops.int/

United Nations Environment Programme (UNEP). (2002). Montreal Protocol on Substances that Deplete the Ozono Layer: Report of the Rigid and Flexible Foams Technical Options Committe. Obtenido de http://www.teap.org

Wright, J. (2003). Environmental Chemistry (First ed.). London: Routledge.

Zhang, H., Kuo, T., Lu, H., \& Huang, S. (1997). Environmentally conscious design and manufacturing: A state-of-the-art survey. Journal of Manufacturing Systems, 352-371. 